\title{
CONOCIMIENTOS, ACTITUDES Y PRÁCTICAS SOBRE HIGIENE BUCAL DE MADRES DE NIÑOS DE 1 A 2 AÑOS QUE FRECUENTAN EL CENTRO DE SALUD DE SANGOLQUÍ, QUITO, ECUADOR
}

\author{
KNOWLEDGE, ATTITUDES AND PRACTICES ON ORAL HYGIENE OF MOTHERS OF \\ CHILDREN FROM 1 TO 2 YEARS FREQUENT TO THE SANGOLQUÍ HEALTH CENTER, \\ QUITO, ECUADOR
}

Jaramillo Nathalia ${ }^{1}$ Núñez Alejandra ${ }^{1}$, Armas Ana del Carmen ${ }^{1 a}$

\section{RESUMEN}

Objetivo: Evaluar el conocimiento sobre salud bucal de madres de niños menores de dos años que acuden a un centro de salud en la ciudad de Sangolquí, cantón Rumiñahui, provincia de Pichincha entre febrero y agosto del 2015 . Material y métodos: 64 madres, seleccionadas de forma aleatoria, tras firmar el consentimiento informado, y verificación de cumplimiento de los criterios de inclusión establecidos, fueron interrogadas sobre conocimientos, prácticas y actitudes frente a la higiene bucal de sus hijos y sus condiciones socioeconómicas. Resultados: El 31,25\% refirió una edad entre los 26 y 30 años. El 35,94\% presentó el estado civil de casada. La instrucción superior en curso fue el nivel más frecuente $(31,25 \%)$. La prueba de Chi cuadrado evidenció que no existe relación entre edad de la madre y nivel de conocimientos $(p=0,198)$, demostrando que existe relación estadísticamente significativa entre los conocimientos sobre higiene bucal de las madres y el grado de instrucción alcanzado por estas $(p=0,015)$. Conclusión: En las condiciones que el estudio fue ejecutado, se puede concluir que las madres de niños menores de dos años que acuden a un centro de salud en la ciudad de Sangolquí, cantón Rumiñahui, provincia de Pichincha, entre febrero y agosto del 2015 posee un nivel regular de conocimientos en cuanto a higiene bucal en sus hijos.

Palabras clave: Conocimientos; higiene bucal; madres; salud bucal. (Fuente: DeCS)

\section{ABSTRACT}

Objective: To evaluate the oral health knowledge of mothers of children under two years of age who attended a health center in the city of Sangolquí, Rumiñahui, province of Pichincha (Ecuador), between February and August 2015. Materials and method: 64 mothers randomly selected, after signing the informed consent and verification of compliance with the established inclusion criteria, were questioned about knowledge, practices and attitudes regarding the oral hygiene of their children and also about their socioeconomic conditions. Results: $31.25 \%$ reported an age between 26 to 30 years, $35.94 \%$ married civil status, current higher education was the most frequent level in $31.25 \%$. The Chi square test showed that there is no correlation between mother's age and knowledge level $(p=0.198)$, showing that there is a statistically significant correlation between mothers' oral hygiene knowledge and the degree of instruction achieved by them $=0.015$ ). Conclusion: Under the conditions of the present study, it can be concluded that mothers of children under two years of age who attend a health center in the city of Sangolquí, Rumiñahui province, province of Pichincha between February and August 2015 have a Regular level of knowledge regarding oral hygiene in their children.

Key words: Health knowledge, oral hygiene, mothers, oral health. (Source: MeSH NLM)

1 Universidad Central del Ecuador -Facultad de Odontología.

${ }^{a} \mathrm{PhD}$ en Operatoria Dental, Docente de Odontología.

\section{Correspondencia:}

Alejandra Núñez

Dirección: Calle Rocafuerte y Ambato. Ambato. Tungurahua- Ecuador

Número telefónico: 593+995749866

Correo electrónico: ale_nu1804@hotmail.es

Citar como: Jaramillo N, Núñez A, Armas A. Conocimientos, actitudes y prácticas sobre higiene bucal de madres de niños de 1 a 2 años que frecuentan el Centro de salud de Sangolquí, Quito, Ecuador. KIRU. 2017; 14(2):52-57. https://doi.org/10.24265/kiru.2017.v14n1.07
Este es un artículo Open Access distribuido bajo la licencia Creative Commons Atribución-NoComercialCompartirlgual 4.0

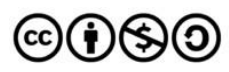




\section{INTRODUCCIÓN}

La Odontología moderna está orientada a la prevención de enfermedades y promoción en salud bucal ${ }^{(1)}$. Las diferentes estrategias desarrolladas por los distintos países buscan garantizar una correcta atención a todo individuo de manera multicultural, promoviendo un servicio eficiente y de excelente calidad de manera equitativa, fomentando el establecimiento de planes estratégicos basados en la promoción de la salud, el diagnóstico precoz y la instauración de tratamientos oportunos ante las principales patologías durante todas las etapas de vida, manteniendo el bienestar físico, mental y social de los individuos ${ }^{(2)}$.

La caries y la enfermedad periodontal son las patologías más comunes que afectan a la cavidad bucal. Con un inicio en los primeros años de vida, presentan un importante incremento con la edad y una prevalencia cada vez mayor en preescolares asociada a sus múltiples factores desencadenantes $^{(3)}$. El fomento de acciones en las prácticas de hábitos saludables y el mantenimiento del control de factores de riesgos más comunes es uno de los aspectos más importantes en la prevención de la caries dental, exigiendo para su control modificaciones importantes en el estilo de vida independiente del nivel sociocultural(4).

Entendiendo que todo cambio en un hábito requiere de esfuerzos ${ }^{(5)}$, en lo referente a salud bucal el profesional odontólogo cumple un papel fundamental en la prevención, como educador, promotor y motivador, evaluando en este proceso los factores de riesgo cariogénicos, los factores sociales inmiscuidos y las características individuales de cada individuo ${ }^{(6)}$. Busca con estos esfuerzos generar hábitos ${ }^{(5)}$. Este cambio de conducta, sin embargo, requiere contar con la predisposición de cada individuo y la colaboración de los padres en el caso de niños en etapa preescolar ${ }^{(7)}$ por las limitaciones propias asociadas a la edad ${ }^{(6)}$.

En países en vías de desarrollo como el Ecuador, pese a los múltiples programas gubernamentales de salud, aún existe influencia directa de creencias ligadas al entorno biopsicosocial ${ }^{(8)}$. En ese contexto, la madre de familia, por su rol de cuidadora permanente del niño desde sus primeras etapas de vida, se convierte en la responsable directa del cuidado de la salud del niño ${ }^{(9)} \mathrm{y}$, desde el punto de vista cariogénico, es transmisora de los microorganismos desencadenantes de procesos cariosos mediante una contaminación pasiva directa o indirectamente a través de alimentos, agua, utensilios, besos ${ }^{(10)}$.

La transmisión vertical es considerada la forma directa de transmisión de los mismos serotipos en los hijos y sus respectivas madres ${ }^{(11)}$. Diferentes estudios genotípicos afirman que dentro de los periodos críticos existe mayor susceptibilidad del individuo para presentar caries como un problema de salud ${ }^{12)}$. Estos periodos se encuentran circunscritos entre los 6 y 24 primeros meses de vida del menor (13), observando que mientras más temprano se produzca la colonización de microorganismos en boca, mayor será el riesgo de desarrollar caries ${ }^{(14)}$. De esta manera, los primeros meses de vida serán claves en el mantenimiento de la salud bucal del menor (15), siendo en este periodo la madre la responsable directa de desarrollar estrategias de cuidado, por ser ella la responsable directa del cuidado del menor en esta etapa de la vida(16). Ella es un elemento transmisor de hábitos y espejo de actitudes para su hijo, que permanecerán por el resto de su vida(17).

Estudios en países desarrollados demuestran una prevalencia de caries en un $12 \%$ que contrasta con el $70 \%$ en países en desarrollo, donde medidas de prevención han sido direccionadas mediante procesos de educación a la madre como responsable de la salud bucal del niño ${ }^{(18)}$. La atención precoz y manutención de la salud bucal antes de completar el primer año de vida se ha convertido en una maniobra preventiva eficiente, acompañada de la limpieza doméstica de la boca, un uso adecuado del biberón, el fomento de la leche materna, acompañada de la prohibición en el consumo de azúcares siempre bajo orientación y asesoramiento nutricional anticariogénico, el uso de fluoruros y una visita temprana dental (19). Todos estos esfuerzos se dirigen hacia las madres por ser ellas las responsables de brindar atención al niño desde sus primeros momentos de vida.

Con estos antecedentes, nuestro estudio pretende evaluar el conocimiento sobre salud bucal de madres de niños menores de dos años que acuden a un centro de salud en la ciudad de Sangolquí, cantón Rumiñahui, provincia de Pichincha ente febrero y agosto del 2015.

\section{MATERIAL Y MÉTODOS}

Se plantea un estudio transversal descriptivo, ejecutado en madres que acuden al área de vacunas en el centro de Salud de Sangolquí, cantón Rumiñahui, provincia de Pichincha, Ecuador, entre febrero y agosto del 2015. Tras autorización del 
Comité de Ética de la Universidad Central del Ecuador y de las autoridades del centro de salud en mención, los objetivos del proyecto fueron presentados y una muestra de 64 madres escogidas por conveniencia, las que una vez explicado el propósito del estudio y su metodología, fueron interrogadas sobre diferentes aspectos para verificar el cumplimiento de los criterios de inclusión establecidos.

Se tomó como base que sean las madres legales del menor, que el niño en cuestión presente entre 12 a 24 meses de vida, ser ellas las cuidadoras directas del menor, encontrarse en capacidad mental de brindar información y no haber sido diagnosticadas con alguna enfermedad psicológica o sistémica incapacitante que impida tomar cuidado del menor, y que el menor no cuente con algún tipo de síndrome o enfermedad sistémica.

Tras la firma de autorización de participación en documento de consentimiento informado, cada participante fue entrevistada sobre conocimiento, actitudes y prácticas en higiene bucal mediante encuesta, la misma que pese a pasar por un proceso previo de validación, encontrarse en idioma español y haber sido aplicada en Perú, fue revisada en cuanto a su idoneidad y concordancia en el idioma, para lo cual fue aplicada en una muestra $n=10$. Se sometió cada pregunta a validación mediante el Alfa de Cronbach $y$ se obtuvo valores de $x=0,70$ considerados satisfactorios, establecida su idoneidad aplicada como instrumento de recolección de información.

A esto se sumaron 9 preguntas tomadas de la encuesta (20) sobre datos socioeconómicos de la madre como fuente directa de la entrega de respuestas. Cada ficha siguió los principios éticos de investigación en seres humanos y fue identificada con códigos preservando la identidad e información proporcionada. Los datos recolectados en las fichas fueron ingresados en programa Excel y luego procesados mediante el paquete estadístico SPSS versión 23.0, analizados mediante la prueba de Chicuadrado, considerando las características socioeconómicas y los conocimientos de higiene bucal.

\section{RESULTADOS}

De las 64 madres que constituyeron la muestra, el $31,25 \%$ refirió una edad de entre 26 a 30 años, presentando el $35,94 \%$ estado civil de casada. La instrucción superior en curso fue el nivel más frecuente en el $31,25 \%$. En cuanto al número de hijos, el 48,44\% manifestó que era su único hijo. Con respecto al número de personas en el hogar, el $34,38 \%$ afirmó contar con hogares constituidos por 4 personas; el 26,56\% manifestó ser jefe de hogar, con una remuneración mensual de más de 2 salarios mínimos (40,63\%), y el $35,94 \%$ contaba con un trabajo independiente. Sobre la condición de la vivienda, el 46,88\% manifestaron habitar una vivienda arrendada.

Con respecto al conocimientos de higiene bucal, tomando como base trabajos previos (Ríos, 2014), el nivel de conocimiento se agrupó en malo, regular y bueno. Los datos socioeconómicos y niveles de conocimiento fueron relacionados mediante la prueba de Chi cuadrado con un $5 \%$ de error, evidenciando que no existe relación entre edad de la madre y nivel de conocimientos $(p=0,198)$, lo que se mantiene con el estado civil $(p=0,352)$, el número de hijos $(p=0,169)$, con el hecho de ser jefe o no de hogar $(p=$ $0,506)$, con su remuneración ( $p=0,529)$, con el hecho de tener vivienda propia, prestada 0 arrendada $(p=0,301)$ y con el tipo de ocupación que desempeñe $(p=0,456)$. Se destaca que existe relación estadísticamente significativa entre los conocimientos sobre higiene bucal de las madres con el grado de instrucción alcanzado por esta $(p=0,015)$ y se demuestra que el conocimiento aumenta de manera proporcional con el nivel de instrucción, resultado que también se refleja al relacionar con el número de personas que viven en el hogar $(p=0,015)$.

\section{DISCUSIÓN}

Entendiendo que la prevención en aspectos de salud se inicia en el hogar ${ }^{(21)}$ y que los problemas de salud varían de acuerdo con la edad del individuo ${ }^{(12)}$, resulta indispensable considerar la importancia de trabajar en procesos educativos preventivos desde la etapa intrauterina ${ }^{(22)}$, afirmación que se refuerza por múltiples estudios que han demostrado que la educación temprana y el establecimiento de hábitos de higiene oral son una de las mejores herramientas de control de problemas de salud a nivel sistémico y bucal.

El contacto que la madre crea influye positivamente en el comportamiento que desarrolla el hijo como individuo dependiente de ella ${ }^{(16)}$; de ahí la importancia de estudios como este, que pretenden conocer el grado de conocimiento, actitudes y prácticas que las madres demuestran sobre temas de salud, para poder desarrollar estrategias sobre cómo mejorarlos o reforzarlos.

Los resultados de este estudio demostraron un bajo conocimiento por parte de las madres sobre la 
higiene bucal de sus hijos, coincidiendo con lo reportado en otros estudios ${ }^{(23)}$; sin embargo, de ello resulta indispensable considerar las limitaciones en cuanto a la muestra evaluada y enfatizar en que el nivel socioeconómico de los participantes de este estudio muestra un grupo perteneciente a la clase media baja, lo que lleva a pensar en la necesidad de ejecutar estudios similares en otros grupos socioeconómicos.

La salud y enfermedad desde hace pocos años atrás pasaron a ser consideradas como resultantes de diferentes factores. En este contexto, los determinantes sociales y económicos cumplen un papel fundamental en estos procesos, por la evidente relación con el acceso a servicios como por el contacto con medidas educativas ${ }^{(24)}$. En los procesos de enfermedad a nivel oral, la presencia de caries y enfermedad periodontal ocupan los puntos de mayor prevalencia ${ }^{(3)}$, de ahí que la gran mayoría de estrategias preventivas se basen en la instauración de medidas de higiene oral ${ }^{(25)}$. El nivel de conocimientos sobre salud oral de las madres se relacionó significativamente con la edad y la ocupación, las actitudes se relacionaron significativamente con el nivel de instrucción, y la práctica en salud oral se relacionó con la ocupación y el estado civil $(p<0,01)$. En nuestro estudio, si bien no fueron consideradas de forma separada estos tres elementos, pudimos evidenciar que el nivel de educación alcanzado por la madre guardó una estrecha relación con el nivel de conocimientos, actitudes y prácticas en general $(p=0,015)$.

El componente socioeconómico en este estudio fue evaluado mediante la encuesta ENEMDU 2007, la cual fue presentada por el INNEN sin modificación alguna. Las respuestas analizadas permitieron determinar que se estaba trabajando con un grupo de ingresos económicos medios bajo, lo que guarda estrecha relación con lo reportado en la literatura que muestra que en el interés por la salud en general guarda relación con los ingresos económicos que el individuo perciba(26). Se destaca que pese a ser frecuente este hecho, no puede convertirse por ningún concepto en un paradigma, ya que la educación determinada por las diferentes estrategias que las entidades de salud desarrollen o las acciones individuales ejecutadas influirán de manera positiva sobre el conocimiento y percepción del individuo para desencadenar cambios en su comportamiento ${ }^{(27)}$.

En el Ecuador, la promoción en salud bucal es ejecutada en centros escolares. Los resultados de este estudio, sin embargo, evidencian la necesidad de una promoción continua, sobre todo enfocada a madres en periodo gestante por la influencia positiva que se presenta en esta etapa sobre la madre y, por tanto, en el niño ${ }^{(28)}$. De ahí la importancia de realizar estudios como este, considerando poblaciones mayores y de otros niveles socioeconómicos.

Entendiendo que la presencia de caries está estrechamente relacionada con la edad, clase social, nivel de higiene, acceso a dispositivos, elementos fluorados 0 el consumo frecuente de hidratos de carbohidratos simples y bebidas azucaradas (27), resulta importante, como odontólogos, la valoración del riesgo del paciente, sin desconsiderar la falta de conocimientos y de buenos hábitos de higiene bucal como punto determinante de la enfermedad ${ }^{(12)}$.

\section{CONCLUSIONES}

En las condiciones que el estudio fue ejecutado, se puede concluir que las madres de niños menores de dos años que acudieron a un centro de salud en la ciudad de Sangolquí, cantón Rumiñahui, provincia de Pichincha, entre febrero y agosto del 2015, poseen un nivel regular de conocimientos en cuanto a higiene bucal de sus hijos.

\section{Contribuciones de autoría}

JN participó en el diseño del estudio, en asesoría técnica, redacción y análisis de resultados, NA participó en la revisión de la literatura, aporte de material de estudio y recolección de datos JN, NA y AA redactaron el artículo el cual fue aprobado por todos los participantes.

\section{Fuente de financiamiento}

Autofinanciado.

\section{Conflictos de interés}

Los autores declaran no tener conflictos de interés en la publicación de este artículo.

\section{REFERENCIAS}

1. Mier $Y$, Rodríguez $M$, Saavedra $E$, Barrios $L$. Conocimiento sobre salud buco-dental de las embarazadas. Consultorio La California. 2012. Gac. Méd Espirit (EEUU). 2013; 15(1): 20-29.

2. Urgélles, Y, Arsenia, M. Algunas consideraciones sobre la alimentación del lactante para la conservación de su salud bucal. Medisan, 2012; 22:596-605.

3. Aguayo C., Abarca M, Aliaga D, Mantileo M, Nass L. Conocimientos, actitudes, conductas y estado de salud oral del adulto mayor del gran Concepción. Revista ANACEO, 2015; 6. 
4. Mairobys, S. Hábitos de higiene bucal y el nivel socioeconómico de niños entre 15 y 20 meses de edad. Acta odontológica Venezolana. 2007; 45(2): 1-2

5. Garbanzo G. Organizational Development and Change Processes in Educational Institutions, a Challenge for the Management of Education. Revista Educación, 2016; 40(1):67-87.

6. González C, Cano M, Meneses E, Saldarriaga V. Oral Health Knowledge of the Caregivers of the 'Buen Comienzo' Program. Revista Gerencia y Políticas de Salud. 2016; 15(31):130-144.

7. González F, Sánchez R., Carmona L. Indicadores de riesgo para la caries dental en niños preescolares de laBoquilla, Revista de salud pública, (Cartagena) 2009; 11(4):620-630.

8. Acevedo, G., Martinez, G., Estario, J. Manual de Salud Pública, Manual of Public Health. Editorial Brujas. 2007. 57.

9. Sánchez C, Rivera R, Correa A, Figueroa M, Sierra A, Izazola S. El desarrollo del niño hasta los 12 meses. Orientaciones al pediatra para su vigilancia con la familia. Acta pediátrica de México. 2015; 36(6):480496.

10. Rotemberg W, Smaisik F. Inmunidad bucal en la primera infancia. Odontoestomatología. 2015; 12(14):414.

11. Chamorro A., Ospina A., Arango J., Martínez C. Acción de la inmunoglobulina secretora en el proceso de adherencia de Streptococcus mutans al diente humano. CES Odont, 2013; 26(2): 76-106.

12. Aguilar $F$, Duarte A, Rejón D, Serrano P, Pinzón D. Prevalencia de caries de la infancia temprana y factores de riesgo asociados. Acta Pediátrica, 2014; 35:259- 266

13. Chandna P, Kuma V. Oral Health in childrenGuidelines for Pediatricians. Springer Link, 2010; 323327.

14. Ojeda J, Oviedo E, Salas A. Streptococcus mutans and dental caries. Ces Odontología, 2013; 26(1):44-56.

15. Quintero G. Estreptococos cariogénicos predominantes, niveles de infección e incidencia de caries en un grupo de escolares. (ADM) 2014; 64(2):45-51.

16. González F. Conocimientos, actitudes y prácticas en salud bucal de padres y cuidadores en hogares infantiles. Medic Od. (Colombia) 2012; 2: 23-35.
17. Araujo A, García C. Conocimientos de los padres sobre la salud bucal de niños preescolares: Desarrollo y validación de un instrumento. Rev. Estomatol. Herediana, 2015; 25(2): 112-121.

18. Manríquez J, Misrachi C, Fajreldin V, Kuwahara K. La clínica del bebé: Una alternativa de salud oral de la población Mapuche-Williche de isla Huapi. Revista Fac. Odontol. Uni. (Antioq). 2014; 342-358.

19. Pachano $\mathrm{S}$, Barrios R. Caries de la infancia temprana según determinantes sociodemográficos, conductuales, nutricionales y relacionados a la transmisión de microorganismos. BOL. ASOC. Argent. Odontp. NIÑOS. 2011; 32(3):8-15

20. ENENDU. Instituto Nacional de Estadística y Censos del Ecuador. Encuesta Nacional de Empleo, Desempleo y Subempleo - Diciembre 2011. Versión 1.0 del conjunto de datos de uso público (enero de 2012), suministrada por el Instituto Nacional de Estadística y Censos del Ecuador.

21. Treviño S. Conocimientos en salud bucal en el entorno social .Revista Médica.2008; 12 (2):232-333.

22. Nuñez W. Caries dental y desarrollo infantil temprano. Revista Chilena de Pediatría Elsevier, 2014; 29(1): 2223-2322.

23. Teixeira G, Vázquez $P$, Domíguez C, Portaluppi V, Alfonzo V, Mao Bernal L. Nivel de conocimientos de madres sobre higiene bucal en niños de 0 a 3 años. Rev. Salud. 2010; 1(1):3-12.

24. Angelino A. Prevención de factores de riesgo: Impacto del ejercicio y los programas de rehabilitación cardiovascular en el riesgo cardiovascular de pacientes coronarios. Revista Médica Clínica Las Condes, 2012; 23(6): 766-771.

25. Wilf R., Frydman K. Inmunidad bucal en la primera infancia. Odontoestomatología, 2010; 12(14): 234-340.

26. Silva $X$. Prevalencia de Caries, gingivitis $y$ maloclusiones en escolares de la ciudad de Victoria, Taumalipas y su relación con el estado nutricional. Revista Odontológica Mexicana (MEX) 2013; 17(4):221-227.

27. Devadas, N., Harikiran A. Effectiveness of oral health education programs. A systematic review. J Int Soc Prev Community Dent, 2013; 3(2):103-115.

28. Ministerio de Salud Pública de Ecuador. MSP. Caries guía práctica clínica. 2015; Obtenido de http://salud.gob.ec. 